\title{
The determination of cetirizine in the presence of cephalosporins through novel RP-HPLC method and application in human serum
}

\author{
H. Shamshad ${ }^{1 *}$, H. Naseem ${ }^{2}$ \\ ${ }^{1}$ Research Institute of Pharmaceutical Sciences, Faculty of Pharmacy, University of Karachi, Karachi, Pakistan. \\ ${ }^{2}$ Department of Pharmaceutical Chemistry, Faculty of Pharmacy, Ziauddin University Karachi, Pakistan.
}

*Corresponding Author's email: henathebest@gmail.com

\begin{abstract}
The current article described a completely validated RP-HPLC method that described simultaneous quantification of cetirizine in the presence of any of the three cephalosporins, i.e., ceftriaxone or cephalexin or cefotaxime while amlodipine (calcium channel blocker) used as an internal standard. Cetirizine, a widely prescribed drug as allergy medication. It is also co-administered with cephalosporins. The methanol: acetonitrile: water in a ratio of 65:5:30 with a $\mathrm{pH}$ to 3.2 with phosphoric acid mobile phase was selected to keep the method least expensive. All the validation parameters such as accuracy, precision, repeatability, suitability were checked and were found to be friendly according to ICH guidelines. The LOD and LOQ in each case were evaluated and for cetirizine were found to be 0.001 and $0.003 \mu \mathrm{gmL}^{-1}$, ceftriaxone 0.06 and $0.211 \mu \mathrm{gmL}^{-1}$, cephalexin 0.016 and $0.054 \mu \mathrm{gmL}^{-1}$, cefotaxime 0.012 and $0.04 \mu \mathrm{gmL}^{-1}$, respectively. The method was found to possess applications for clinical purposes and pharmaceutical labs.
\end{abstract}

Keywords: Cetirizine, ceftriaxone, cephalexin, cefotaxime, amlodipine, RP-HPLC.

\section{INTRODUCTION}

Cetirizine, a second-generation H1-receptor antagonist, has been safely administered with cephalosporins [1-2]. However, an exceptional example of contact-type urticaria ensuing from obtained sensitization to cephalosporin compounds [3]. In another study, the administration of different cephalosporins in penicillin-allergic patients was related to a considerable boost in the pace of sensitivity to reactions. Several HPLC methods have been reported for cephalosporins. An HPLC method was described to analyze a broad range of cephalosporin congeners, with simply three reagents for extraction and drug analysis. However, the mobile phase was complexed and consisted of different proportions of $0.01 \mathrm{M}$ sodium acetate with acetonitrile and methanol [4]. Laurence [5] developed an HPLC method to determine chloramphenicol and five cephalosporin antibiotics. In this method, acetate buffer, up to $70 \%$, was used as the mobile phase. Yasufuku et al. [6] applied the HPLC method to determine residual cephalosporins in a beef meet. The recoveries were at levels of 78.3 to 91.6\%. Arayne et al. [7] developed the RP-HPLC method to estimate cefpirome in the presence of essential and trace metal. Shinde et al. [8] developed an RP-HPLC method for the simultaneous determination of cephalexin and probenecid in the tablets. The minimum detection limit obtained for both the drugs was two $\mu \mathrm{gmL}^{-1}$. In this method, nifedipine was used as an internal standard. Similarly, several HPLC methods of cetirizine have been reported in the literature. Zarapkar et al. [9] developed a simple, fast, and precise RP HPLC method for the simultaneous determination of cetirizine and pseudoephedrine in tablets. Another RP-HPLC method for the simultaneous analysis of three drugs viz phenylpropanolamine $\mathrm{HCl}$, cetirizine dihydrochloride, and acetaminophen from a dosage form was presented by Kanumula et al. [10] and used aspirin as an internal standard. The separation was achieved on an Inertsil C8 (5 $\mu, 250 \mathrm{x}$ $4.6 \mathrm{~mm})$ with a mobile phase of water, acetonitrile, and triethylamine (60:40:0.1 v/v/v). In another method, cetirizine was quantified using diazepam as an internal standard. The separation was performed at $13 \mathrm{mM}$ phosphoric acid solution and acetonitrile (61:39 v/v) as a mobile phase [11]. Walily et al. [12] determined a simple HPLC assay of cetirizine in the presence of one of its synthetic precursors (hydroxyzine $\mathrm{HCl}$ ) at a flow rate of $2 \mathrm{mLmin}^{-1}$. To determine simultaneously (+) and (-) cetirizine in human urine, Choi and collaborators developed a chiral separation method using HPLC. The mobile phase consisted of phosphate buffer and acetonitrile for urine assays [13]. Jelinska et. al., [14] and co-workers developed and validated another HPLC method to determine cetirizine in tablets using a LiChrosorb RP-18 column with a mobile phase $\mathrm{KH}_{2} \mathrm{PO}_{4}$ and acetonitrile in $65: 35$ ratio. A flow rate of $2 \mathrm{mLmin}^{-1}$ at $230 \mathrm{~nm}$ with methylparaben as an internal standard was used. Sher et al., [15] determined antiallergic and antihistaminic drugs, including cetirizine, domperidone, chlorphenamine maleate, loratadine, meclizine, and buclizine. However, no report was available for the simultaneous determination of cetirizine with ceftriaxone or cephalexin, or cefotaxime was developed. Arayne et al., [16] developed an accompanying method to determine cefpirome and cetirizine or levocetirizine in human plasma. However, in this method, $75 \%$ of acetonitrile in the mobile phase was used, which was very expensive for the routine analysis of drugs. Our group determined cetirizine with $\mathrm{H}_{2}$ receptor antagonists [17] and statins drugs [18]. Hence, an economical approach was adopted in the present work to develop a method for determining these drugs for routine analysis and clinical labs.

\section{EXPERIMENTAL}

\subsection{Materials}


Pharmaceutical grade of cetirizine dihydrochloride, ceftriaxone, cephalexin, cefotaxime reference standards were obtained from various pharmaceutical industries. HPLC grade methanol was bought from Merck Germany.

\subsection{Instrumentation}

The HPLC instrument consisted of an LC-10 AT VP pump, SPD-10A VP UV-vis detector (Shimadzu Japan). A Purospher ${ }^{\circledR}$ STAR RP-18 end-capped column $(5 \mu \mathrm{m}, 25$ x $0.46 \mathrm{~cm})$ was used. Class-GC 10 software (Shimadzu Japan) was used for the acquisition of data.

Mobile phase preparation

The mobile phase consisted of a mixture of two organic solvents and water (methanol: acetonitrile: water, 65:5:30), and $\mathrm{pH}$ was adjusted to 3.2 with orthophosphoric acid.

Preparation of solutions

An equivalent amount of $10 \mathrm{mg}$ of cetirizine and cephalosporin standards were accurately weighed in a $100 \mathrm{~mL}$ volumetric flask and filled up to the mark with the mobile phase. This standard solution was filtered and injected into the system. An aliquot of this solution was accordingly diluted with methanol to get a final concentration of $2-50 \mu \mathrm{gmL}^{-1}$ for cetirizine and cephalosporin. A fixed concentration of internal standard, i.e., $25 \mu \mathrm{gmL}^{-1}$, was added in each of these dilutions. These sets were kept in the refrigerator to inject further for three days.

\subsection{Validation procedures}

All the attributes for the validation purpose were performed according to the guidelines set by the International Conference on Harmonization [19].

\subsection{System suitability, specificity, and selectivity}

The six replicates of a standard solution were injected into the system, and each injection response was performed. Theoretical counts and resolution parameters were also studied for the method.

\subsection{Linearity, accuracy, and precision}

Linearity studies were performed on $50,25,20,15,5$, and $2 \mu \mathrm{gmL}^{-1}$ concentrations for all the drugs and constructed the calibration curve. Four representative samples achieved precision on every three days, while accuracy was evaluated at concentrations of $8,10,12 \mu \mathrm{gmL}^{-1}$ for the three drugs $(\mathrm{n}=6)$ by spiking method.

\subsection{Ruggedness}

The solution was allowed to stand at ambient temperature for 3days and then analyzed against the freshly prepared standard. Moreover, method ruggedness was also performed by changes in the slight $\mathrm{pH}$ of the solution and calculating the \%RSD in each case.

\section{Robustness}

Robustness was performed by performing the same analysis set by different analysts and comparing the result, and calculating \% RSD in each case.

Serum drug analysis

The stated chromatographic conditions determined the recovery studies of cetirizine and cephalosporins in human serum. $10 \mathrm{~mL}$ of blood was deproteinated by acetonitrile, filtered using a 0.45 -micron membrane filter, and was mixed to different aliquots to made concentrations in the range of $2-50 \mu \mathrm{gmL}^{-1}$.

\section{RESULTS AND DISCUSSION}

\subsection{Optimization}

Initially, the peaks of cetirizine and cephalosporins could not be separated using different concentrations of methanol and water. A small quantity of acetonitrile was also added systematically for completely sharp peak shapes in this costeffective method. The mixture of methanol: acetonitrile: water in a ratio of 65:5:30 with a $\mathrm{pH}$ adjusted to 3.2 was capable of a reasonable separation and short runtime. The detection was performed at $230 \mathrm{~nm}$ using amlodipine as an internal standard eluting out at 4.5 minutes. The retention times of each cephalosporin, i.e., cephalexin, ceftriaxone, and cefotaxime, were found to be 3 minutes, and that of cetirizine was found to be 5.5 minutes.

\subsection{System Suitability}

Theoretical plate count assessed system suitability and resolution parameters for cephalosporins and cetirizine were found to be 2059 and $2339,2.39$, and 4.25 , respectively.

\subsection{Selectivity and Linearity}

The chromatogram acquired was identical to the standard and spiked solution with an equivalent concentration. The representative chromatograms (Figure) showed no other peaks on the retention time of cetirizine and cephalosporins; the retention times did not change. The correlation coefficients (Table 1) determined for all the drugs demonstrated excellent linearity in the range of $2-50 \mu \mathrm{gmL}^{-1}$.
Ceftriaxone
Cephalexin
$\mathrm{y}=60856 \mathrm{x}-66123$
$\mathrm{y}=11909 \mathrm{x}+59194$
Cefotaxime
$y=17322 x+19892$ 
Cetirizine

$$
y=13923 x+65717
$$

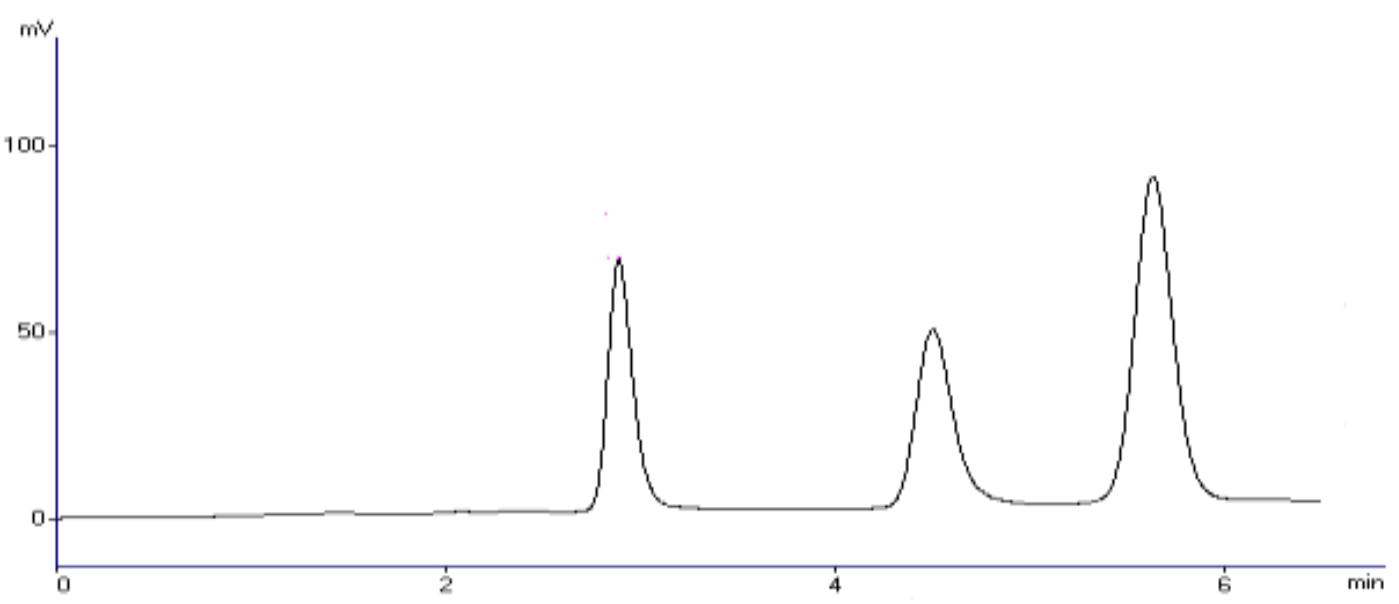

Figure: Representative chromatogram of cephalosporin, internal standard and cetirizine.

Table 1: Regression statistics and sensitivity of the method

\begin{tabular}{|l|c|c|c|}
\hline \multicolumn{1}{|c|}{ Drugs } & $\mathrm{R}^{2}$ & $\begin{array}{c}\mathrm{LOQ} \\
\mu \mathrm{gmL}^{-1}\end{array}$ & $\begin{array}{c}\text { LOD } \\
\mu \mathrm{gmL}^{-1}\end{array}$ \\
\hline Ceftriaxone & 0.999 & 0.211 & 0.06 \\
\hline Cephalexin & 0.999 & 0.054 & 0.016 \\
\hline Cefotaxime & 0.999 & 0.04 & 0.012 \\
\hline Cetirizine & 0.999 & 0.003 & 0.001 \\
\hline
\end{tabular}

\subsection{Sensitivity}

The method's sensitivity was evaluated (Table 1) by determining the LOD and LOQ for all the drugs assayed.

\subsection{Accuracy and precision}

Accuracy and precision were determined six times per concentration (Table 2). Within- and between-day precision values are given in Table 3 using the \%recoveries and \%RSD values.

Table 2: Accuracy and precision of the method

\begin{tabular}{|l|c|c|c|}
\hline \multirow{3}{*}{ Drugs } & $\begin{array}{c}\text { Conc. } \\
\%\end{array}$ & $\%$ RSD & \%Recovered \\
\hline \multirow{3}{*}{ Ceftriaxone } & 80 & 0.94 & 99.83 \\
\cline { 2 - 4 } & 100 & 0.28 & 99.71 \\
\cline { 2 - 4 } Cephalexin & 120 & 0.45 & 100.3 \\
\cline { 2 - 4 } & 80 & 0.44 & 99.68 \\
\cline { 2 - 4 } & 100 & 0.61 & 99.76 \\
\cline { 2 - 4 } Cefotaxime & 120 & 0.13 & 99.15 \\
\cline { 2 - 4 } & 80 & 1.06 & 100.56 \\
\cline { 2 - 4 } Cetirizine & 100 & 0.23 & 99.83 \\
\cline { 2 - 4 } & 120 & 0.14 & 100.51 \\
\cline { 2 - 4 } & 80 & 1.15 & 100.49 \\
\cline { 2 - 4 } & 100 & 0.34 & 100.23 \\
\hline
\end{tabular}

\subsection{Robustness and ruggedness}

The robustness and ruggedness of the present work were determined at average, and changing a few analytical conditions like wavelength, mobile phase composition, flow rate, instrument, and analyst, and \%RSD was found less than $\pm 2 \%$ in all parameters.

\subsection{Serum samples}

The method was further subjected to the analysis in serum (Table 3). The method was originated to be appropriate for the remedial point. 
Table 3: Intermediate precision of the method (\%RSD)

\begin{tabular}{|c|c|c|c|c|c|c|c|c|c|c|c|c|c|c|c|c|}
\hline Conc & \multicolumn{4}{|c|}{ Ceftriaxone } & \multicolumn{4}{c|}{ Cephalexin } & \multicolumn{4}{c|}{ Cefotaxime } & \multicolumn{4}{c|}{ Cetirizine } \\
\hline $\begin{array}{c}\mu \mathrm{gmL}^{-} \\
1\end{array}$ & Day1 & Day2 & Day3 & Day4 & Day1 & Day2 & Day3 & Day4 & Day1 & Day2 & Day3 & Day4 & Day1 & Day2 & Day3 & Day4 \\
\hline 2 & 0.81 & 0.02 & 0.99 & 1.09 & 0.08 & 0.42 & 0.66 & 1.11 & 1.7 & 0.98 & 1.71 & 1.22 & 1.11 & 0.97 & 1.88 & 1.21 \\
\hline 5 & 2.33 & 2.68 & 0.36 & 0.99 & 0.66 & 0.39 & 0.84 & 1.26 & 1.23 & 1.26 & 1.02 & 1.54 & 0.36 & 0.48 & 0.98 & 1.08 \\
\hline 15 & 0.036 & 0.24 & 0.74 & 1.12 & 0.58 & 1.09 & 0.9 & 0.95 & 1.71 & 0.69 & 0.88 & 0.89 & 0.69 & 0.72 & 0.61 & 0.84 \\
\hline 20 & 0.023 & 0.18 & 0.92 & 1.31 & 0.96 & 1.35 & 0.99 & 0.86 & 0.99 & 1.03 & 0.71 & 0.93 & 0.72 & 0.79 & 0.71 & 0.76 \\
\hline 25 & 0.07 & 0.47 & 0.63 & 0.97 & 0.87 & 0.87 & 1.09 & 0.74 & 0.06 & 1.18 & 0.85 & 0.97 & 0.81 & 0.86 & 0.85 & 0.94 \\
\hline
\end{tabular}

\section{CONCLUSION}

In the present work, a high quantity method was developed for the first time to determine cetirizine in the presence of ceftriaxone or cephalexin, or cefotaxime. The technique did not involve expensive instrumentation or excessive use of costly organic solvents or corrosive column solvents. The method was found to have wide application in pharmaceutical evaluation or for clinical purposes.

\section{CONFLICT OF INTEREST}

No potential conflict of interest relevant to this article was reported.

\section{ACKNOWLEDGMENTS}

The author is thankful to Dr. Agha Zeeshan Mirza, Umm Al Qura University, for his help with the manuscript

\section{REFERENCES}

1. Sultana N., Arayne MS., Shamshad H., Mirza AZ, Naz MA., Fatima B., Asif M, Mesaik M. A., (2011). Spectroscopy 26 (4-5), 317-328.

2. Apotex.com (http://www.apotex.com/ca/en/products/privatelabel/Cetirizine.asp)

3. Louis T, (1975). Arch Dermatol, 111, 1609.

4. Steven AS, Thomas MF and James ST, (1984). Antimicrobial Agents and Chemotherapy, 26(5), 652-655.

5. Laurence AD, (1983). Clinical Chemistry, 29(5), 856-858

6. Yasufuku K, Makiyama I, Fuchimoto A and Oshima H., (1999). Journal of the Japan Veterinary Medical Association, 52(5), 321-325

7. Arayne MS, Sultana N and Nawaz M, (2006). Pakistan Journal of Pharmaceutical Sciences, 19(1), 39-44.

8. Shinde VM, desai BS and Tendolkar NM, (1994). Indian Journal of Pharmaceutical Sciences, 56(2), 58-60.

9. Zarapkar SS, Halkar UP and Rane SH, (1998). Indian drugs, 35 (10), 658-661.

10. Kanumula GV, Bhanu R and Sunderesan M, (2001). Indian Drugs, 38(6), 294-298.

11. Zaater MF, Tahboub YR and Najib NM, (2000) J. Pharm. Biomed. Anal., 22(5), 739-744.

12. Walily El AFM, Korany MA, Gindy El A and Bedair MF, (1998). J. Pharm. Biomed. Anal., 17(3), 435-442.

13. Choi SO, Lee SH, Hak S, Kim EJ and Choo HYP, (2000). Arch Pharmacal research, 23(2), 178-181.

14. Jelinska A., Stanisz B., Zajac M., Musial W and Ostrowicz A, (2000). Acta poloniae pharmaceutica 57(3), 171173.

15. Sher N., Siddiqui FA., Hasan N., Shafi N., Zubair A., Mirza AZ., (2014). Analytical Methods, 6(8), 2704-2714.

16. Arayne MS, Sultana N and Nawaz M, (2008). Journal of Analytical chemistry, 63(9), 881-887.

17. Sultana N., Arayne MS., Shamshad H., (2010). Medicinal Chemistry Research, 19 (5), 462-474.

18. Shamshad H., Sultana N., Arayne MS., (2015). Analytical Chemistry Letters, 5 (2), 109-11.

19. ICH, Topic Q2A, Guideline, I. H. T. (2005). Q2 (R1), 1(20), 05. Methodology, PMP/ICH/281/95. 